\title{
A Large-Scale Open Motion Dataset (KFall) and Benchmark Algorithms for Detecting Pre-impact Fall of the Elderly Using Wearable Inertial Sensors
}

\author{
Xiaoqun Yu, Jaehyuk Jang and Shuping Xiong* \\ Department of Industrial and Systems Engineering, College of Engineering, Korea Advanced Institute of Science and \\ Technology (KAIST), Daejeon, South Korea
}

Research on pre-impact fall detection with wearable inertial sensors (detecting fall accidents prior to body-ground impacts) has grown rapidly in the past decade due to its great potential for developing an on-demand fall-related injury prevention system. However, most researchers use their own datasets to develop fall detection algorithms and rarely make these datasets publicly available, which poses a challenge to fairly evaluate the performance of different algorithms on a common basis. Even though some open datasets have been established recently, most of them are impractical for pre-impact fall detection due to the lack of temporal labels for fall time and limited types of motions. In order to overcome these limitations, in this study, we proposed and publicly

OPEN ACCESS

Edited by:

Nina Skjæret-Maroni, Norwegian University of Science and

Technology, Norway

Reviewed by:

Rogier Landman, Broad Institute, United States

Eduardo Casilari,

University of Malaga, Spain

*Correspondence:

Shuping Xiong

shupingx@kaist.ac.kr

Received: 09 April 2021 Accepted: 15 June 2021 Published: 16 July 2021

Citation:

Yu X, Jang J and Xiong S (2021) A Large-Scale Open Motion Dataset (KFall) and Benchmark Algorithms for Detecting Pre-impact Fall of the Elderly Using Wearable Inertial Sensors.

Front. Aging Neurosci. 13:692865. doi: 10.3389/fnagi.2021.692865 provided a large-scale motion dataset called "KFall," which was developed from 32 Korean participants while wearing an inertial sensor on the low back and performing 21 types of activities of daily living and 15 types of simulated falls. In addition, ready-to-use temporal labels of the fall time based on synchronized motion videos were published along with the dataset. Those enhancements make KFall the first public dataset suitable for pre-impact fall detection, not just for post-fall detection. Importantly, we have also developed three different types of latest algorithms (threshold based, support-vector machine, and deep learning), using the KFall dataset for pre-impact fall detection so that researchers and practitioners can flexibly choose the corresponding algorithm. Deep learning algorithm achieved both high overall accuracy and balanced sensitivity (99.32\%) and specificity (99.01\%) for pre-impact fall detection. Support vector machine also demonstrated a good performance with a sensitivity of $99.77 \%$ and specificity of $94.87 \%$. However, the threshold-based algorithm showed relatively poor results, especially the specificity (83.43\%) was much lower than the sensitivity (95.50\%). The performance of these algorithms could be regarded as a benchmark for further development of better algorithms with this new dataset. This large-scale motion dataset and benchmark algorithms could provide researchers and practitioners with valuable data and references to develop new technologies and strategies for pre-impact fall detection and proactive injury prevention for the elderly.

Keywords: pre-impact fall, fall detection, public dataset, wearable sensor, algorithm development 


\section{INTRODUCTION}

The safety and health of old people have increasingly drawn attention due to accelerated global population aging. Falling is a serious problem faced by our society as $28-35 \%$ of the population aged 65 or older suffer at least one fall per year (Organization et al., 2008), and $20-30 \%$ of fall accidents lead to mild to severe injuries or even death (Lord et al., 2007). In order to mitigate the serious consequences of falls, multiple studies have been conducted to develop fall detection systems.

Based on the types of sensors being used, fall detection systems can be divided into context-aware systems and wearable systems. Context-aware systems mainly rely on ambient sensors, such as radar and floor sensors as well as vision-based devices (Igual et al., 2013). One fundamental disadvantage of such systems is that they are restricted to indoor use, so they cannot detect the fall anywhere and anytime. In fact, up to 50\% of the falls happen outside home premises (Lord et al., 2007). Over the past decade, wearable inertial sensor-based fall detection systems have gained tremendous popularity among researchers because they offer high portability (no space constraints), accurate motion sensing, and low cost (Micucci et al., 2017). Therefore, this study particularly focuses on wearable inertial sensors. Generally, there are two main directions for the development of wearable inertial sensor-based fall detection systems. The majority of existing studies focus on post-fall detection, which is designed to rapidly detect fall events and initiate medical alarms timely to reduce the frequency and severity of long lies (Aziz et al., 2017). However, this approach has an inherent drawback, that is, it cannot prevent fall-induced injuries since fall impacts have already occurred. Another branch of studies targets pre-impact fall detection, which aims to detect the fall during the falling period but before body-ground impact. Therefore, it could activate on-demand fall protection systems, such as wearable airbags, to prevent injuries caused by the fall impact ( $\mathrm{Hu}$ and $\mathrm{Qu}, 2016)$. This method provides a more fundamental solution for the elderly for fall injury prevention. However, it is also more challenging than post-fall detection because the sensor signal of body-ground impact moment, which includes most differentiated information (usually with peak acceleration and angular velocity), cannot be seen by algorithms.

In recent years, researchers have begun to shift their focus from post-fall detection to pre-impact fall detection and shed some light on this topic. Jung et al. (2020) developed a threshold-based algorithm, which combined multiple thresholds (magnitude of acceleration, magnitude of angular velocity, and vertical angle) based on inertial sensors for pre-impact fall detection and achieved $100 \%$ sensitivity and $97.54 \%$ specificity with an average lead time of $280 \mathrm{~ms}$. This algorithm was developed based on their own simulated dataset with six types of falls and 14 types of activities of daily living (ADLs) by 30 young subjects. Another research conducted by Kim et al. (2019) applied seven machine learning algorithms and two deep learning algorithms to detect pre-impact fall, using accelerometers, and most of the models achieved $\geq 98 \%$ sensitivity and specificity. Similarly, those algorithms were based on their own dataset with 10 types of falls and 14 types of ADLs by 12 subjects. Quite recently, one group of researchers has proposed a multisource $\mathrm{CNN}$ ensemble framework for pre-impact fall detection based on the data from four pressure sensors, one acceleration sensor, and one gyro sensor (Wang et al., 2020). Ten subjects participated in their experiment, and each subject performed four types of falls and five types of ADLs. This deep learning architecture also reached high accuracy of $99.30 \%$, with an average lead time of $350 \mathrm{~ms}$. Even though the reported results were impressive, earlier studies only showed good performances of the developed algorithms on their relatively small datasets (small number of human subjects and limited types of motions), and they rarely made those datasets publicly available. This poses a challenge to fairly evaluate the performance of different algorithms on a common basis and their generalizability to different datasets. A few preliminary studies showed that algorithms based on a specific database with good performance had poor external validity on other databases (Sabatini et al., 2015; Jung et al., 2020). For instance, when Jung et al. (2020) applied their thresholds to the SisFall dataset (Sucerquia et al., 2017), both sensitivity and specificity dropped considerably by 4 and $7 \%$, respectively. Similarly, Bourke et al. (2008) proposed an algorithm, using the vertical velocity of the trunk as the threshold and achieved $100 \%$ sensitivity and specificity on a dataset, which was built from five subjects with four types of falls and six types of ADLs. However, the same threshold with optimized value only yielded $80 \%$ sensitivity on a comparatively larger dataset with five types of falls and seven types of ADLs acquired from 25 subjects (Sabatini et al., 2015). The lack of public datasets also makes it hard to objectively compare newly developed algorithms (Noury et al., 2008). This situation thus hinders the technology advancement for pre-impact fall detection, which is expected to protect the elderly from fall injuries in a proactive way.

Some public fall databases, such as SisFall, tFall, MobiFall, and FallAllD, have been established recently. However, they are only appropriate for post-fall detection rather than pre-impact fall detection. The details will be discussed in the next section. To overcome the aforementioned limitations, in this study, we proposed and publicly provided a large-scale motion dataset called KFall. This dataset is expected to be the first public dataset suitable for pre-impact fall detection, not just for postfall detection. We also developed three benchmark algorithms, using this new dataset, which allows researchers to fairly compare their new algorithms for pre-impact fall detection. This largescale motion dataset and benchmark algorithms could provide researchers and practitioners with valuable data and reference to develop new technologies and strategies for pre-impact fall detection and injury prevention for the elderly.

\section{RELATED PUBLIC FALL DATASETS}

As mentioned in the introduction, due to the limitations of context-aware systems, the review of public fall datasets emphasized on wearable inertial sensors and was carried out through five major electronic databases (Scopus, ScienceDirect, IEEE Explorer, Web of Science, and Google Scholar). Two basic inclusion criteria were utilized for refining the search results: 
(1) datasets should be fully open to the public and published in English; (2) there should be at least 10 subjects in the datasets. In addition, a recent review paper, which performed a comprehensive analysis of public datasets for wearable fall detection systems, was also referred (Casilari et al., 2017a). The same group of authors has reviewed public datasets again very recently and applied CNN to those datasets for fall detection (Casilari et al., 2020). Cross-check was implemented to prevent missing any important references for this study. In the end, our search yielded 16 representative datasets (Table $\mathbf{1}$ ).

As illustrated in Table 1, no dataset provides temporal labels for the fall time, which annotates the fall onset moment (when a fall begins) and the fall impact moment (when the body hits the ground) in the sensor data sequence. Lack of temporal labels for the fall time will not influence the development of algorithms for post-fall detection, since sensor data in the fall impact moment has very distinguishable patterns, which are usually with a peak value of acceleration and angular velocity. However, for preimpact fall detection, it is important to detect the fall during the body descending period but before the moment of bodyground impact. Because of this, the algorithm for pre-impact fall detection should learn to recognize the difference of sensor data between the non-falling period and the falling period based on the known dataset. Therefore, the falling period of the sensor data, which starts from the fall onset moment and ends at the fall impact moment, should be labeled out. Unlike the fall impact moment, without temporal labels or synchronized video clips published together with motion datasets, it is almost impossible to determine the fall onset moment merely by referring to the sensor data since there is no significant signal change from preceding normal activities to the start of falls. Even though the UP-Fall dataset, the CMDFALL dataset, and the TST Fall dataset also released synchronized video references, they were at a low frequency of $18 \mathrm{~Hz}, 20 \mathrm{~Hz}$, and $30 \mathrm{~Hz}$, respectively. Since the entire duration of common falls is very short, with an average interval of $746 \mathrm{~ms}$ (Tao and Yun, 2017), such low frequency would introduce high errors when labeling the fall onset and impact moments.

Another common drawback of publicly available datasets is that most of them include limited types of falls and ADLs $(\leq 10$, e.g., DLR, tFall, MobiFall, Cogent Labs, TST Fall, MobiAct, UMAFall, UniMiB SHAR, IMUFD, CMDFALL, CGU-BES, DU$\mathrm{MD}$, and UP-Fall) to represent complex real-life scenarios. The number of human subjects used to build the dataset is also relatively small $(\leq 20$, e.g., DLR, tFall, TST Fall, Ericiyes University, UMAFall, IMUFD, CGU-BES, DU-MD, UP-Fall, and FallAllD). In addition, we also noticed that orientation data, which represent rich information of human motion (Incel, 2015), were frequently missing among the published datasets except MobiFall and MobiAct.

\section{KFALL DATASET CONSTRUCTION}

We set four main requirements when designing the KFall dataset in order to complement the deficiencies of existing public datasets. (1) High-frequency synchronized video clips should be captured for labeling the fall time of sensor data; (2) the dataset should include various types of falls and ADLs with a sufficient number of subjects; (3) sensor orientation measurement should also be provided, which allows more flexibility to the interested researchers when designing their algorithms; and (4) the sampling frequency and measurement range of the inertial sensor should be sufficient (Saleh et al., 2021).

\section{Data Acquisition System and Experimental Setup}

In order to record the sensor data together with the synchronized high-frequency video clips, a custom data acquisition system was designed. This system can be easily replicated since all the components are available from the market at affordable prices. A nine-axis inertial sensor (LPMS-B2, LP-RESEARCH Inc., Tokyo, Japan), which includes a three-axis accelerometer $( \pm 16 \mathrm{G})$, a three-axis gyroscope $\left( \pm 2,000^{\circ} / \mathrm{s}\right)$, and a three-axis magnetometer $( \pm 16 \mathrm{G})$, was used for collecting motion data. Orientation measurement (Euler angle: roll, pitch, and yaw) provided by the manufacturer is the integration of angular velocity and further modified, using an extended Kalman filter by combining the information from the accelerometer and the magnetometer (Petersen, 2020). The sensor was configured at a frequency of $100 \mathrm{~Hz}$, which was consistent with many studies for pre-impact fall detection (Zhao et al., 2012; Wu et al., 2019). The sensor data were transmitted through Bluetooth Dongle, which was connected to Raspberry Pi 4 (4 GB) as the host PC. As for synchronous video capture, a Raspberry Pi HQ camera mounted with 6-mm 3MP Wide Angle Lens, was used at the maximum FPS of 90. Data acquisition of this research was implemented on a selfdeveloped GUI program, running in Raspberry Pi 4 in which data synchronization between the sensor and the camera was handled by the multiprocessing technique in the Python language.

The inertial sensor was attached to the low back of each subject (see Figure 1A), which was used by many researchers for fall detection (Kwolek and Kepski, 2014; Özdemir, 2016). In order to capture in-depth information of human motion, which is critical to judge the fall onset moment, the camera was set in the front side of the main experiment area rather than directly ahead of it. The whole experimental setup is shown in Figure 1B.

\section{Participants and Experimental Protocol}

This dataset was generated from 32 young Korean males (age: $24.9 \pm 3.7$ years; height: $174.0 \pm 6.3 \mathrm{~cm}$; weight: $69.3 \pm 9.5 \mathrm{~kg}$ ). All of them were healthy and independent, and none of them reported a recent history of musculoskeletal disorder, which could affect their mobility. Every participant signed informed consent for the experimental protocol, which was approved by KAIST Institutional Review Board (IRB No: KH2020-068).

The experimental tasks in our dataset were majorly formulated from the existing public datasets (Casilari et al., 2017a). Since the SisFall dataset has the largest type of falls and ADLs and motions in other datasets were usually a subset of it, the majority of motions in our dataset were directly adopted from SisFall. All types of falls and ADLs in the SisFall dataset were chosen based on a large-scale survey among 15 independent elderly people and 17 retirement homes. For 
TABLE 1 | Wearable inertial sensor-based public datasets for fall detection.

\begin{tabular}{|c|c|c|c|c|}
\hline Public dataset & Types of ADLs/falls & Subjects No. & Sensor data type & Temporal labels for the fall time \\
\hline DLR Frank et al. (2010) & $15 / 1$ & 19 & ${ }^{\S} \mathrm{A}, \mathrm{G}, \mathrm{M}$ & No \\
\hline tFall Medrano et al. (2014) & Not typified/8 & 10 & A & No \\
\hline MobiFall Vavoulas et al. (2014) & $9 / 4$ & 24 & $\mathrm{~A}, \mathrm{G}, \mathrm{O}$ & No \\
\hline Cogent labs Ojetola et al. (2015) & $8 / 6$ & 42 & $A, G$ & No \\
\hline TST fall Gasparrini et al. (2015) & $4 / 4$ & 11 & A & No \\
\hline MobiAct Vavoulas et al. (2016) & $9 / 4$ & 57 & $\mathrm{~A}, \mathrm{G}, \mathrm{O}$ & No \\
\hline Erciyes University Özdemir (2016) & $16 / 20$ & 14 & ${ }^{\S} \mathrm{A}, \mathrm{G}, \mathrm{M}$ & No \\
\hline UMAFall Casilari et al. (2017b) & $8 / 3$ & 17 & ${ }^{\S} \mathrm{A}, \mathrm{G}, \mathrm{M}$ & No \\
\hline SisFall Sucerquia et al. (2017) & $19 / 15$ & 38 & $A, G$ & No \\
\hline UniMiB SHAR Micucci et al. (2017) & $9 / 8$ & 30 & A & No \\
\hline IMUFD Aziz et al. (2017) & $8 / 7$ & 10 & ${ }^{\S} \mathrm{A}, \mathrm{G}, \mathrm{M}$ & No \\
\hline CMDFALL Tran et al. (2018) & $12 / 8$ & 50 & A & No \\
\hline CGU-BES Wang et al. (2018) & $8 / 4$ & 15 & $A, G$ & No \\
\hline DU-MD Saha et al. (2018) & $8 / 2$ & 10 & A & No \\
\hline UP-Fall Martínez-Villaseñor et al. (2019) & $6 / 5$ & 17 & $A, G$ & No \\
\hline FallAIID Saleh et al. (2021) & ${ }^{*} 44 / 35$ & 15 & ${ }^{\S} \mathrm{A}, \mathrm{G}, \mathrm{M}, \mathrm{B}$ & No \\
\hline KFall (Our dataset) & $21 / 15$ & 32 & $\mathrm{~A}, \mathrm{G}, \mathrm{O}$ & Yes \\
\hline
\end{tabular}

A, accelerometer; $G$, gyroscope; $O$, orientation measurement; $M$, magnetometer; $B$, barometer.

$\S$ Complex sensor fusion algorithm should be further applied to obtain the orientation measurement.

"For the same type of a fall, the authors considered all possible directions (left, right, forward, backward) under two conditions (with and without recovery); 12 ADLs were hand motions, and they separated one cyclic ADL into two, such as sit down and stand up.
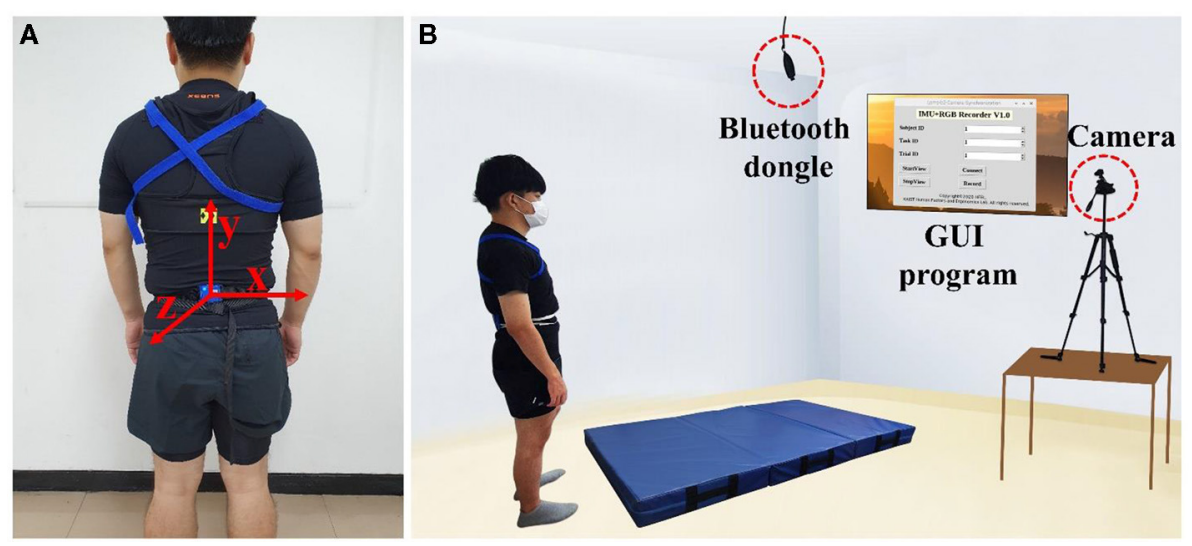

FIGURE 1 | (A) Inertial sensor location and 3D coordinate system; (B) experimental setup.

the ADLs, SisFall covers from simple daily movements (such as walking, sit to stand) to high-dynamic activities (jogging, jumping) and even near-fall scenarios (such as stumbling during walking, collapsing to a chair). As for the falls, we can divide them into three categories based on preceding activities: a fall from walking (such as caused by a slip, a trip), a fall from sitting (such as caused by fainting, trying to get up), and a fall from standing (such as trying to sit down). All the fall activities in the SisFall dataset include preceding ADLs, which are closer to the real-world falls. Another reason for choosing SisFall as our major reference is that it also provides instruction videos of each $\mathrm{ADL}$ and falls so that we can easily reproduce those motions.
Aside from the activities adopted from SisFall, we further added four common static activities, including sitting on a chair, sitting on a sofa, standing, and lying. Those static motions could be used as calibration postures for some fall detection algorithms (Yu et al., 2017). Considering the practical use of this dataset for the elderly population in Korea, two frequently observed ADLs in daily life of older Korean were also newly introduced. They are sitting to the ground and getting up (usually happened in restaurants), and sitting to a sofa with inclining to the back support and getting up (usually happened at home). For these reasons and the data from the Korean participants, we named our dataset as KFall. All fall activities in the KFall dataset are the same as the SisFall. While for several specific ADLs included 
TABLE 2 | Experimental tasks of 21 types of ADLs and 15 types of falls.

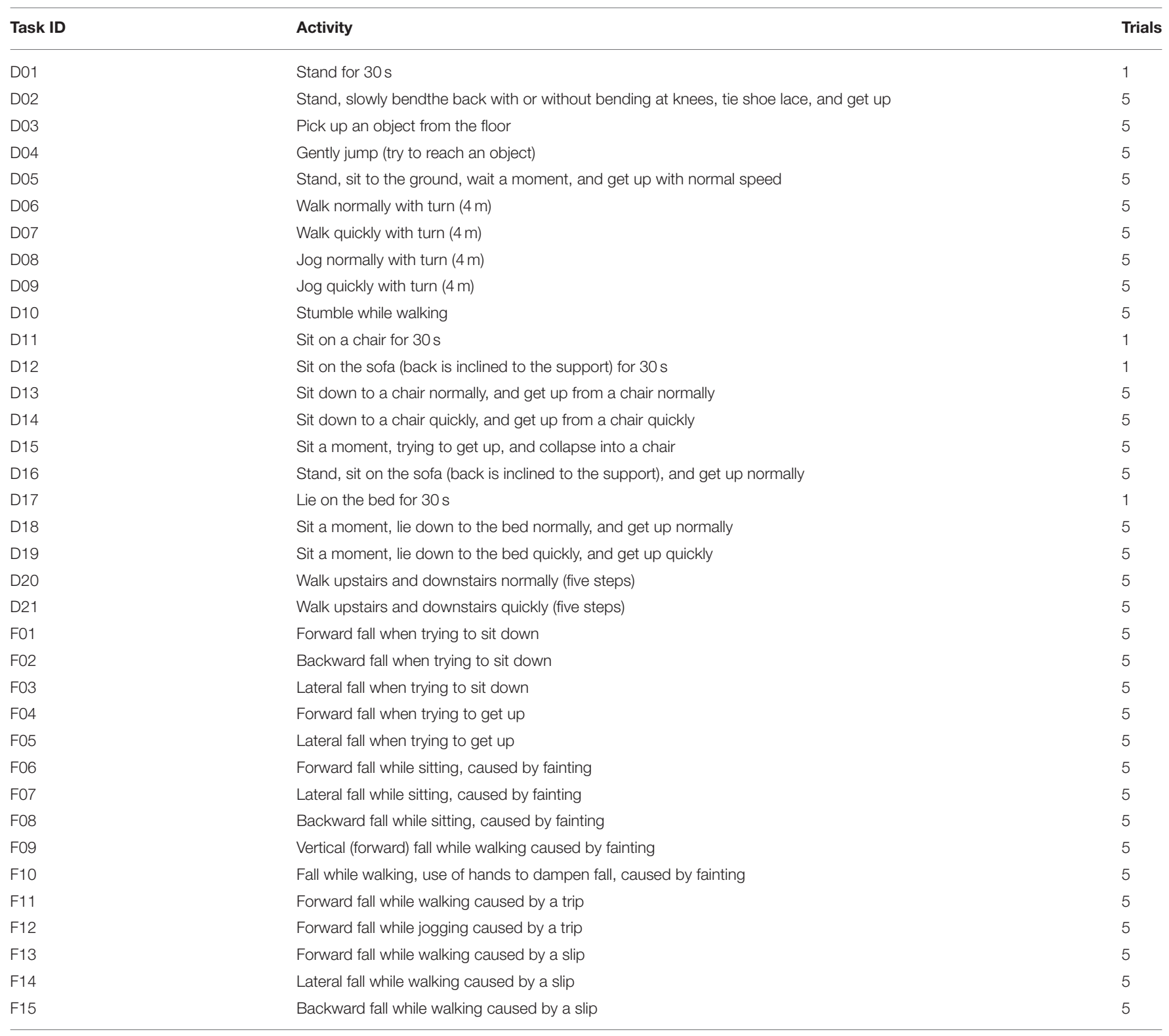

in SisFall, we made some modifications to avoid duplication or make them more natural. For the two motions, which are sitting in a low height chair and getting up slowly or quickly, we used the motion of sitting to the ground and getting up with normal speed (D05) to replace them since they have similar motion patterns. Likewise, with regard to two motions which are standing, slowly bending with or without bending knees and getting up, tying shoelaces (D02) with or without bending at knees, and getting up are the substitutes. We also removed two ADLs considered in the SisFall dataset. One is to rotate the body when lying in a bed since it usually occurs during sleep. Another one is to get into and out of a car due to the facility constraint. Finally, 21 types of ADLs and 15 types of simulated falls were included in the KFall dataset (Table 2). Except for the static tasks (D01, D11, D12, and D17), which required only one trial, all other tasks were designed for five trials. During the experiment, for the ADLs, the subjects were instructed to perform them based on their daily habits to make those motions as natural as possible. While for the fall activities, since the young subjects usually do not have fall experience, instruction videos from the SisFall and on-site demos by experimenters were provided if necessary. To ensure the subject safety, all the fall activities were performed on a 15$\mathrm{cm}$-thick mattress. After the experiment, incomplete data caused by Bluetooth signal disconnections or synchronization errors were removed. Finally, KFall contains a total of 5,075 motion files, including 2,729 ADL motions and 2,346 fall motions. 
F17: FOrward fall while walking caused by a trip

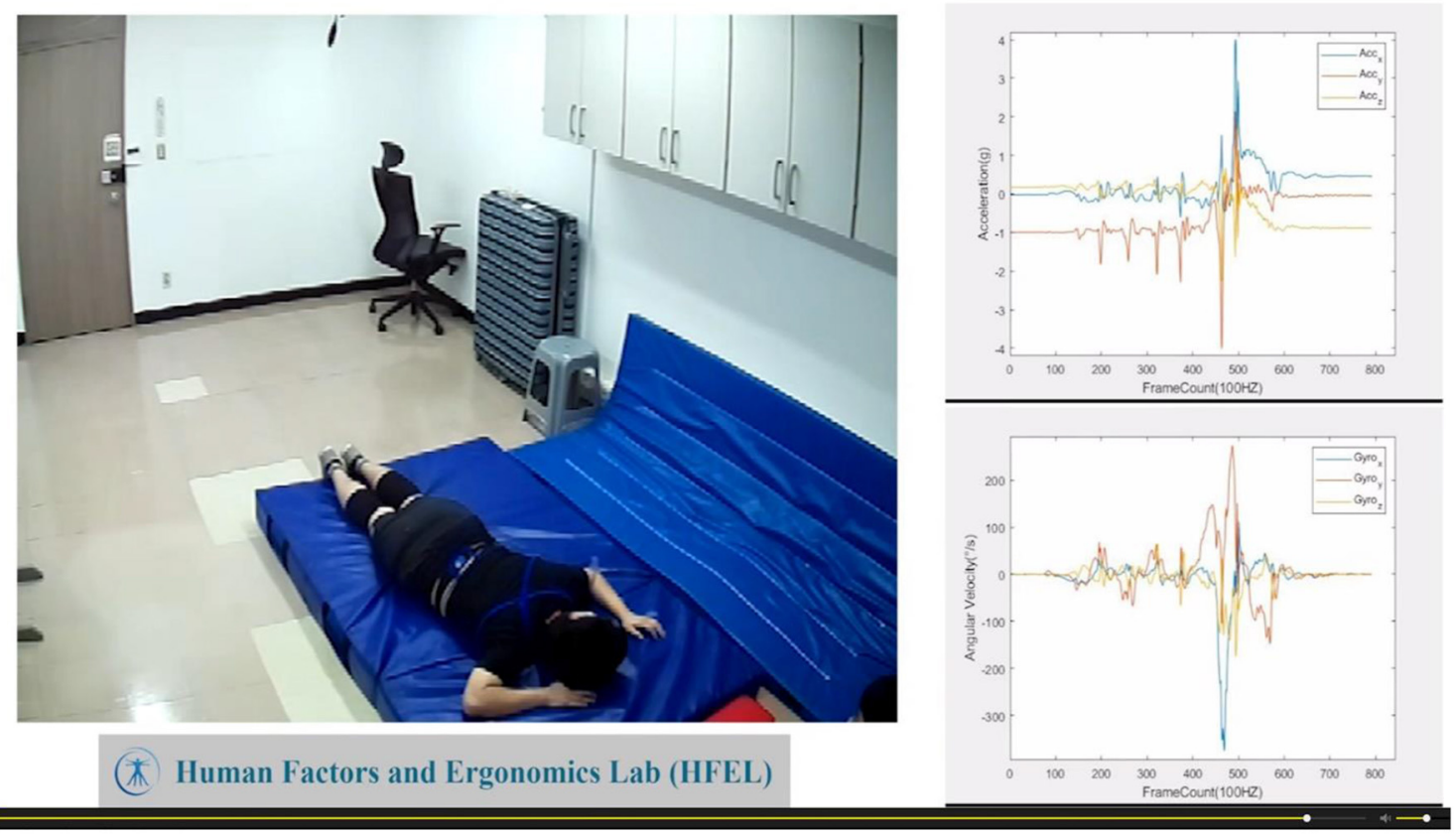

FIGURE 2 | Integrated motion video for the fall time labeling. Left: synchronized fall motion video from the camera; right: sensor data video converted from readings of the inertial sensor (acceleration and angular velocity).

\section{Data Labeling for Fall Time}

Since the existing public fall datasets lack synchronized video references on fall activities, it is very difficult to reliably label the fall onset moment solely based on the sensor data for preimpact detection. On the other hand, because naked eyes cannot recognize subtle motions in the graphics, only referring to the video will also introduce large errors in the labeling process. Therefore, we propose a new method that combines information from both sensor and video data to reduce the labeling error. We firstly converted the sensor data in a csv format into a video format (avi, $100 \mathrm{~Hz}$ ) and further integrated with a fall motion video $(90 \mathrm{~Hz})$ from a Raspberry HQ camera as a whole video, which was played at $90 \mathrm{~Hz}$ (see Figure 2). The video from the sensor data and the camera maintained the same frequency as the original data format to avoid time distortion. Secondly, based on the integrated video, we played synchronized fall motion and sensor data video frame by frame to accurately label the fall onset and fall impact moments.

The fall impact moment can be easily determined since body-ground impact and an acceleration peak are obvious in the integrated video. Whereas, for the fall onset moment, there is a less obvious motion pattern, and it is hard to define it quantitatively. For this reason, we have introduced a semiautomatic method for labeling the fall onset moment after a comprehensive review of the integrated fall videos (see Figure 3). Since the fall is preceded by dynamic movements (e.g., walking, getting up, and sitting down), the $y$-axis of acceleration is usually considered as a sensitive axis. This is because the acceleration on the $y$-axis shows the most obvious pattern during falling. Falls usually have significant motion changes in the gravity direction, which can be detected by the $y$-axis acceleration. However, for the falls caused by fainting during sitting, they are less dynamic and usually without obvious acceleration change at the beginning of falling. Therefore, the $y$-axis of acceleration could not be regarded as the sensitive axis. In such cases, the $\mathrm{x}$-axis or $\mathrm{z}$-axis of the angular velocity can be considered as the sensitive axis because there are more significant changes during rotational movements along the sagittal plane (a forward/backward fall) or the frontal plane (a lateral fall). Those body motion changes during different fall activities (Bourke et al., 2010) reflect local peaks in sensor signals along the sensitive axis. Based on the synchronized fall motion video, we can quickly move the cursor along the timeline to the rough period of the fall onset. Then we evaluated local peaks in the corresponding period of the sensor data video one by one. Those local peaks could be regarded as potential candidates of the fall onset moment. Choosing the local peak among the candidates involves some subjective judgments of the evaluator. Since the proposed semiautomatic labeling method achieved a high degree of consistency in labeling the fall onset moments between the two independent evaluators in the pilot test, the KFall dataset was labeled by an experienced evaluator for time efficiency. One representative case of labeling is illustrated in Figure 4, which is a forward fall during walking caused by a slip. Based on the fall onset and the fall impact moments, the fall event 


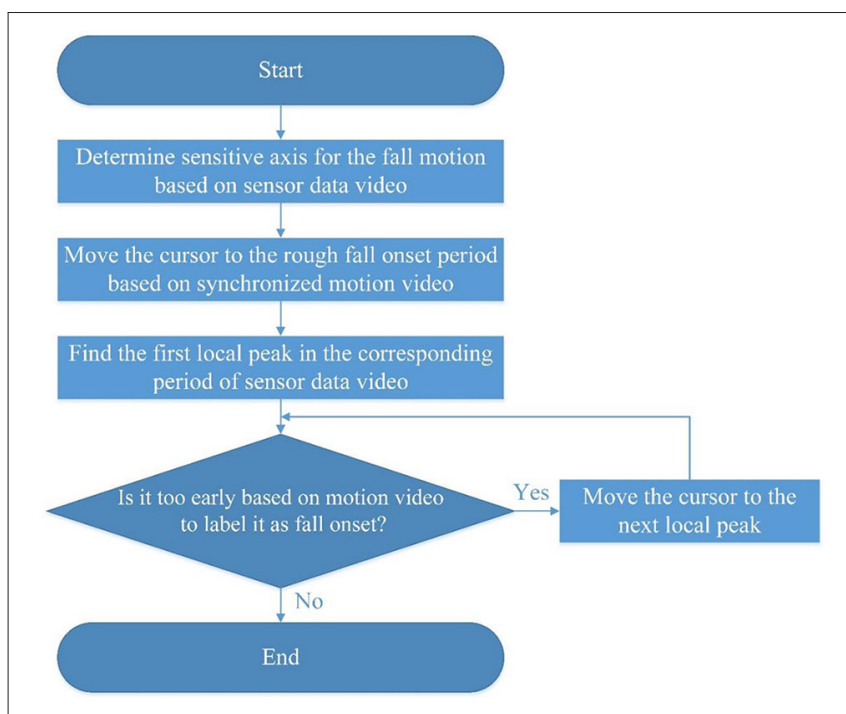

FIGURE 3 | A flowchart for labeling the fall onset moment in sensor data.

could be further divided into three phases: pre-fall, falling, and post-fall phases. Since our major focus is to detect the fall before body-ground impact (a pre-impact fall), the post-fall phase is not considered for the following algorithm development.

All the sensor data, labels of fall trials, and demo videos are publicly available from the Google site: https://sites.google.com/ view/kfalldataset. The detailed data organization is summarized in Figure 5. For each motion file (csv), it contains 11 columns, which are TimeStamp(s), FrameCounter, acceleration (unit: g), angular velocity (unit: ${ }^{\circ} / \mathrm{s}$ ), and Euler angle $\left({ }^{\circ}\right)$ along three axes. Each label file (xlsx) includes temporal labels for the fall time of all the fall trials from each subject, and it has six columns: task code (task ID), description, trial ID, fall onset frame, and fall impact frame in the sensor data. All the demo videos can be accessed from the attached links.

\section{BENCHMARK ALGORITHMS FOR PRE-IMPACT FALL DETECTION}

Based on our newly developed motion dataset KFall, we further developed and tested three different types of algorithms for preimpact fall detection. Those algorithms cover three major distinct categories in the literature: (i) threshold-based (ii) conventional machine learning, and (iii) deep learning algorithms.

For the threshold-based algorithm, four thresholds (magnitude of acceleration, pitch angle, roll angle, and vertical velocity) were considered to detect a pre-impact fall based on recent publications (Jung et al., 2020; Kim et al., 2020). The magnitude of the acceleration is the L-2 norm of acceleration readings from three axes. Pitch and roll angles are defined as the rotations around the $\mathrm{X}$-axis and $\mathrm{Z}$-axis of the sensor. As for the vertical velocity, it is calculated by integrating the vertical acceleration, which is obtained by the Euler angle transformation of the three-axis acceleration data (Lee et al., 2014). The optimal threshold values were determined by the grid search method. Finally, the threshold values of the magnitude of acceleration $\left(\mathrm{ACC}_{\mathrm{M}}\right)$, pitch angle, roll angle, and vertical velocity $(\mathrm{VV})$ were set to $0.8 \mathrm{~g}, 25^{\circ}, 25^{\circ}$, and $0.3 \mathrm{~m} / \mathrm{s}$, respectively. Figure 6 shows the flowchart of the threshold-based algorithm for detecting pre-impact falls.

For the conventional machine learning algorithm, support vector machine (SVM) was applied in this study since it usually achieved better performance in similar tasks (Aziz et al., 2017; Qiu et al., 2018). A comprehensive set of motion features, which encompassed acceleration, angular velocity, and orientationbased information in both temporal and frequency domains, was selected (Incel, 2015). The magnitude of acceleration and angular velocity of each sliding window, with the width of 50 frames $(0.5 \mathrm{~s})$ were utilized for extracting features from the acceleration and angular velocity data (Aziz et al., 2014). Basic features include mean, variance, and root mean square (RMS). More advanced ones are listed as follows: (1) zero-crossing rate (ZCR): the number of samples, which is over the mean of the window; (2) absolute difference (ABSDIFF): the sum of the absolute difference between each sample and the mean of the window divided by the number of samples; (3) First 5-FFT coefficients: the first five of the fast Fourier transform (FFT) coefficients; (4) spectral energy (SE): the sum of the squared FFT coefficients divided by the number of samples. With respect to the orientation-based features, they were calculated from the pitch, roll, and yaw angles. Likewise, mean, standard deviation, RMS, ZCR, ABSDIFF, and SE were derived from those angles. Finally, a total of 40 features were generated and further normalized as the input for the machine learning model.

For the deep learning algorithm, a novel hybrid architecture, integrating both convolution and long short-term memory (ConvLSTM, Figure 7) was adopted from our latest publication (Yu et al., 2020). The model consists of three sequential convolutional blocks followed by two long short-term memory (LSTM) cells with dropout operations and one fully connection layer with softmax activation. Each convolutional block contains operations of convolution, batch normalization, relu, and max pooling. The preceding convolutional layers were designed as automatic feature extractors to provide abstract representations of the sensor raw data in the feature maps. Those high-level features with short-term dependencies were further processed by the recurrent layers, which could capture the long-term temporal relationship of the motion data. Nine-dimensional sensor raw data (three-axis acceleration, three-axis angular velocity, and three Euler angles), with a window size of 50 frames $(0.5 \mathrm{~s})$, were the input of the ConvLSTM model.

Following the general guideline, $80 \%$ of data ( 26 subjects) were randomly chosen as a training set, and the rest $20 \%$ of data (six subjects) were treated for testing purposes. Sensitivity, specificity, and lead time were calculated to evaluate the performance of three different algorithms. Lead time was defined as the time interval between the fall detection moment (when a fall was detected by the algorithm) and the fall impact moment. Sufficient lead time is an important practical application requirement for algorithms to be deployed to on-demand fall protection systems (such as wearable airbags). Algorithm sensitivity and specificity 


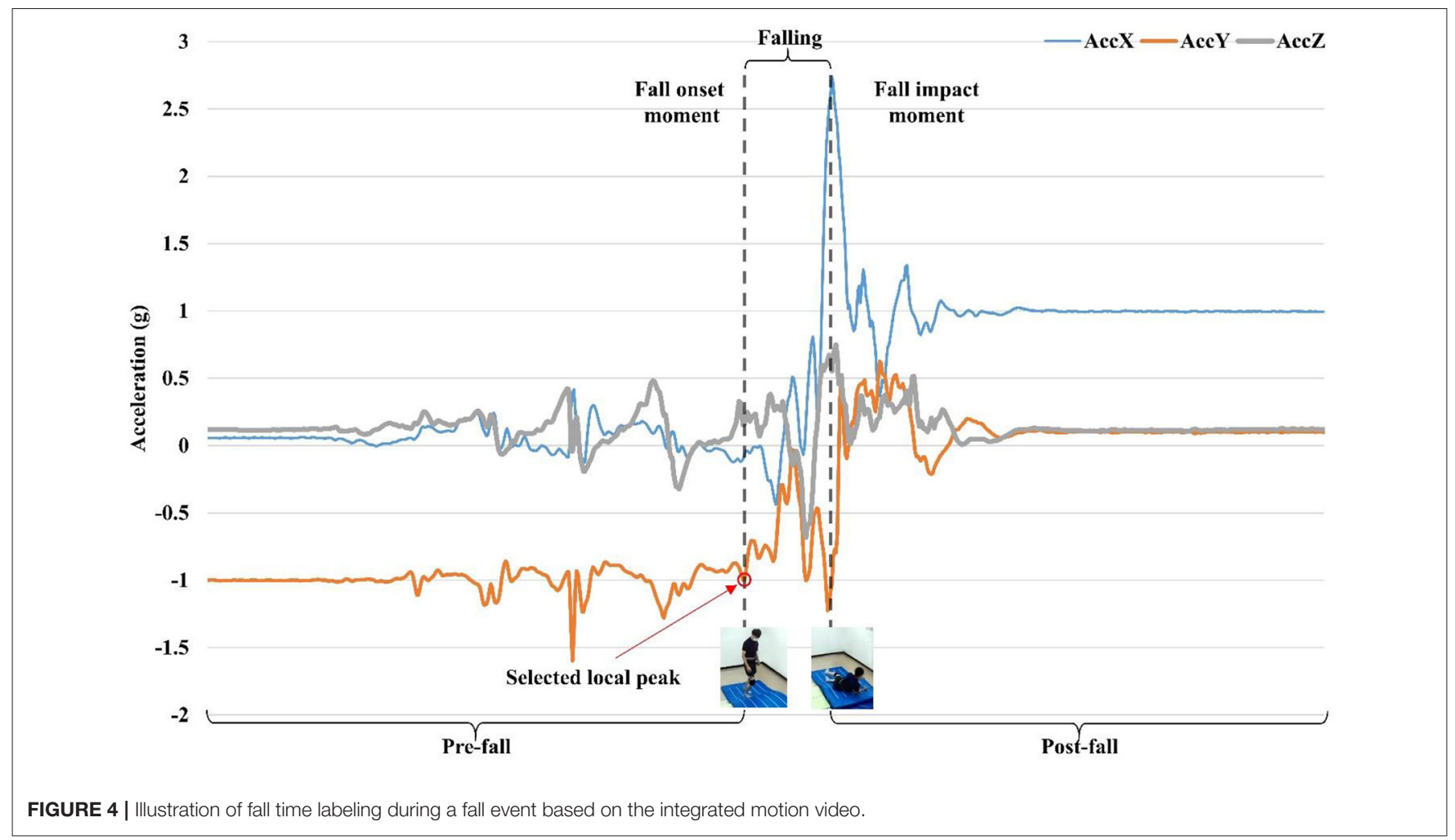

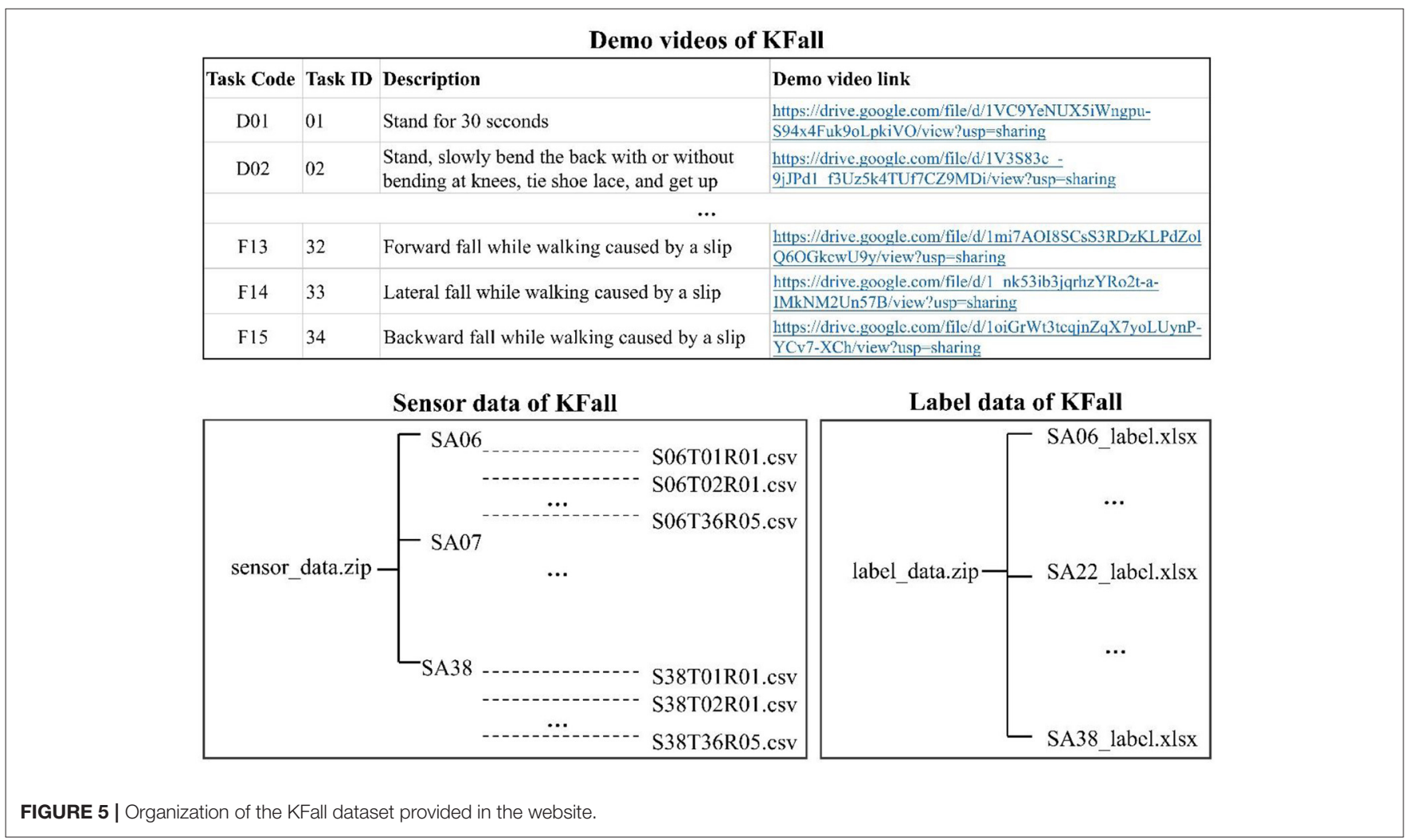




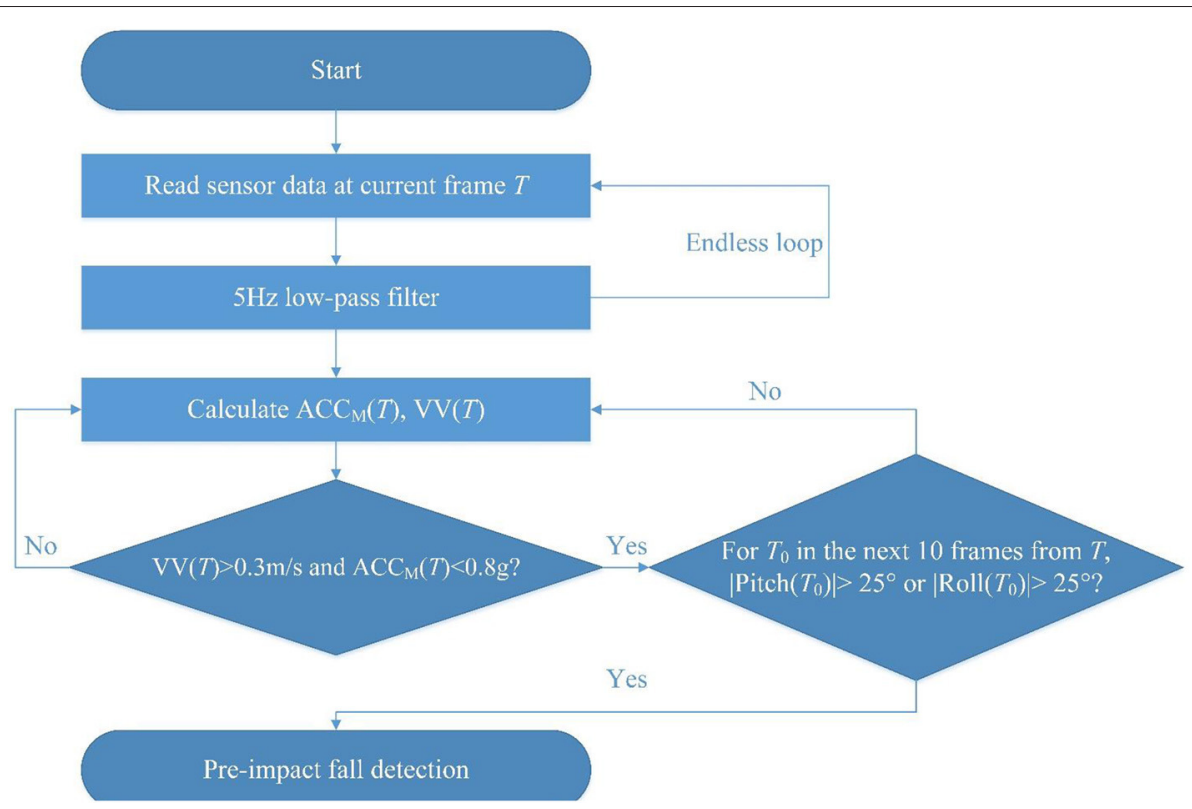

FIGURE 6 | A flowchart of the threshold-based algorithm for pre-impact fall detection.

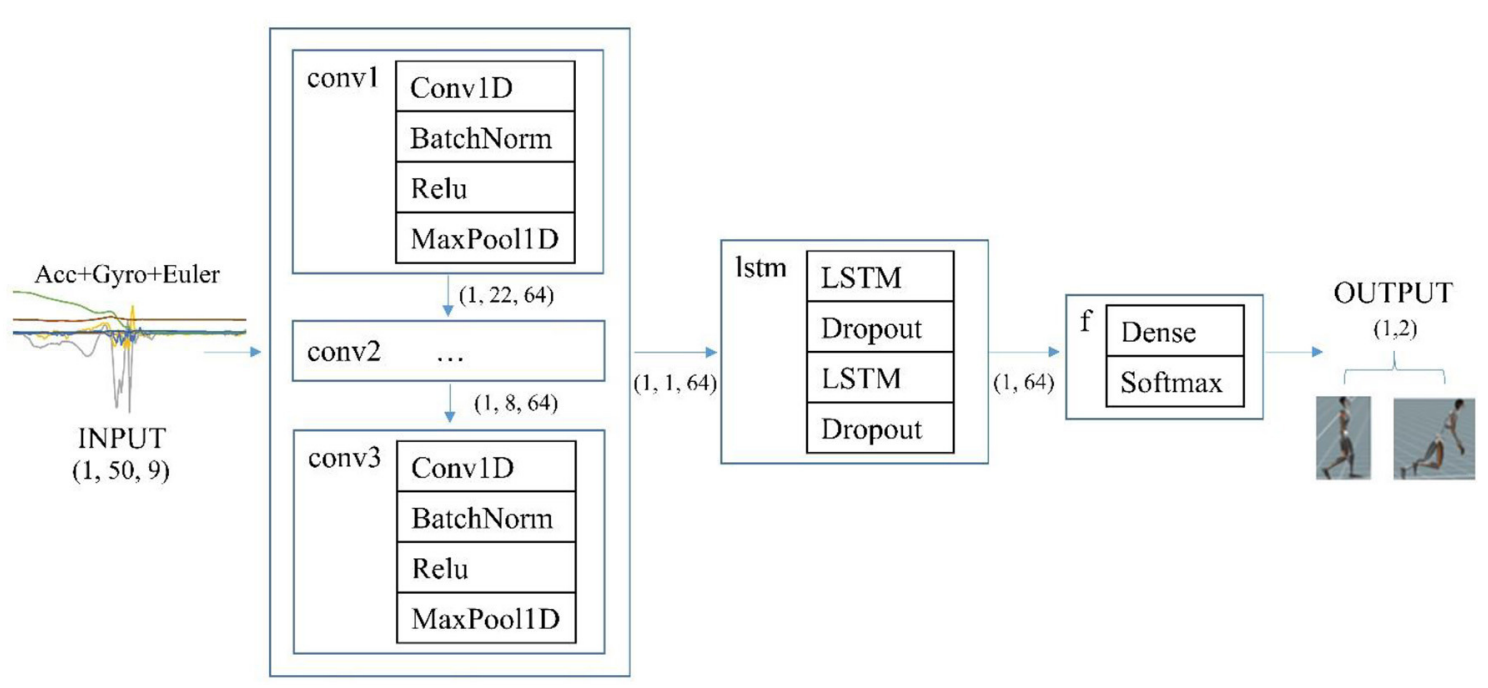

FIGURE 7 | The architecture of the deep learning model (ConvLSTM).

were calculated by equations 1 and 2 , respectively.

$$
\begin{aligned}
& \text { Senvitivity }=\frac{T P}{T P+F N} \\
& \text { Specificity }=\frac{T N}{T N+F P}
\end{aligned}
$$

where TP (true positive) is the number of fall files detected as falls; FN (false negative) is the number of fall files detected as ADLs; $\mathrm{TN}$ (true negative) is the number of ADL files detected as ADLs; FP (false positive) is the number of ADL files detected as falls.
Table 3 shows the overall performance of three different algorithms on the testing set, which contains 444 fall files and $507 \mathrm{ADL}$ files. In terms of accuracy-related measures, conventional machine learning algorithm (SVM) and deep learning algorithm (ConvLSTM) outperformed the thresholdbased algorithm. Particularly, ConvLSTM achieved both high overall accuracy and balanced sensitivity $(99.32 \%)$ and specificity (99.01\%). SVM also had a good performance with sensitivity of $99.77 \%$ and specificity of $94.87 \%$. However, the threshold-based algorithm showed relatively poor results, especially the specificity $(83.43 \%)$ was much lower than the sensitivity $(95.50 \%)$. With 
respect to lead time, ConvSLTM obtained the best performance with an average lead time of $403 \pm 163 \mathrm{~ms}$, which was slightly longer than SVM $(385 \pm 159 \mathrm{~ms})$ but much longer than the threshold-based algorithm $(333 \pm 160 \mathrm{~ms})$.

\section{ALGORITHM VALIDATION ON REAL-WORLD FALL DATASET (FARSEEING)}

In order to further evaluate the feasibility of applying trained algorithms based on our simulated fall dataset of young volunteers to detect real-world falls in the elderly, the FARSEEING dataset, currently, the largest real fall repository (Klenk et al., 2016; Chen et al., 2017), was used in this study. A total of 22 records of real-world falls are available upon request. Each fall file contains 1,200s of data, including data signals of ADLs and falling. In this study, 15 falls were selected because they were collected from the sensors with the same location and data acquisition frequency as our KFall. Those 15 falls were collected from eight older adults (two males, six females; age: $66.9 \pm 6.5$ years; height: $162.2 \pm 9.3 \mathrm{~cm}$; weight: $74.2 \pm 10.3 \mathrm{~kg}$ ). Since half of fall samples lack angular velocity data and they all lack orientation data, in order to fully utilize this dataset, only acceleration data were used. The best model (ConvLSTM) among three benchmark algorithms was selected for the validation purposes. Without changing the network structure, it was retrained only based on the acceleration data from KFall. Since the FARSEEING dataset does not have a video reference, the fall onset moment is defined as $1 \mathrm{~s}$ prior to the fall impact moment as in other studies (Shi et al., 2012; Chen et al., 2017). The data before the fall onset moment were segmented as an ADL file, and the data between the fall onset moment and the fall impact moment were segmented as a fall file, resulting in 15 ADL files and 15 fall files. The data after the fall impact moment were not considered for pre-impact fall detection as we did in the KFall. The same window size of 50 frames $(0.5 \mathrm{~s})$ was also applied. The performance of ConvLSTM on the FARSEEING dataset is summarized in Table 4. The results showed that the ConvLSTM model achieved a sensitivity of $93.33 \%(=14 / 15)$, a specificity of $73.33 \%(=11 / 15)$, and an averaged lead time of $411 \mathrm{~ms}$.

\section{DISCUSSION}

Pre-impact fall detection based on wearable inertial sensors is still a research problem to be solved. Most of papers only published algorithms, using their own datasets but rarely made them publicly accessible, which hinders the development of pre-impact fall detection and proactive injury prevention. So far, there is no open fall dataset suitable for pre-impact fall detection; therefore, we newly established the KFall dataset and made it publicly available for the first time. This motion dataset was developed from the 32 Korean participants while performing 21 types of ADLs and 15 types of falls. This dataset covers almost all typical daily activities and falls, and it is expected to provide researchers and practitioners with a common foundation to develop new algorithms and technologies on pre-impact fall detection and proactive injury prevention.

Compared with the existing public fall datasets (Casilari et al., 2017a; Saleh et al., 2021) in the literature, the biggest advantage of the KFall dataset is that it is constructed with the synchronized video reference and motion sensor data. This enables accurate temporal labels for the fall time and allows the dataset to be further used for pre-impact fall detection, not just post-fall detection. We also proposed a new and semiautomatic method for reliably labeling the fall onset moment by checking local peaks of the sensor data through the integrated motion and sensor data video (Figures 2, 3). Even though this method still involves some subjective judgments from human evaluators, the induced variations should be minimal because the video is at a high frame rate $(90 \mathrm{~Hz})$. Musci et al. (2020) conducted an interesting study for pre-impact fall detection based on the SisFall dataset. Due to the lack of video references in the SisFall dataset, the authors and their colleagues formed an expert panel to label the fall time only based on the sensor data pattern. This approach is very difficult to implement and tends to be less accurate, especially for some falls with very short intervals, such as a backward fall when trying to sit down. In order to maintain the privacy of the participants, the synchronized videos are not open to the public, whereas ready-to-use labels of each fall trial (a fall onset frame and a fall impact frame) are published together with the KFall dataset. Another strength of this dataset is that it contains a comparable number of motion types and human subjects as the three most comprehensive datasets (SisFall, Erciyes University, and FallAllD) in the literature (Table 1). It covers different physical levels of ADLs from low-activity behaviors to highdynamics and even near-fall scenarios, and also covers from lessintensive falls (such as caused by fainting) to very dynamic falls (such as caused by a slip or a trip). This dataset is closer to the complex real-world scenarios, so it is more valuable for research and development in the field of pre-impact fall detection and proactive injury prevention.

In addition, three different types of algorithms for pre-impact fall detection were implemented based on this comprehensive motion dataset. All of them were adopted from the state-ofthe-art algorithms published recently (Jung et al., 2020; Kim et al., 2020; Yu et al., 2020, 2021) and thus were representative to be the benchmarks. It was expected that the thresholdbased algorithm showed poorer performance compared with machine learning algorithms (SVM and ConvLSTM) since the number of motion features considered for the threshold-based algorithm was much less than the other two algorithms. It is usually infeasible to include many thresholds for threshold-based algorithms, which would dramatically increase the searching space and introduce undermined results (e.g., one fall, which satisfies some thresholds, could be against other thresholds). With respect to machine learning algorithms, ConvLSTM had a more balanced sensitivity and specificity (99.32 and 99.01\%) than SVM (99.77 and 94.87\%). Compared with hand-crafted features used in SVM, the automatic features generated by well-designed deep learning neural networks had more distinguishing power of ADLs and falls (Wang et al., 2019). As for the lead time, it is a critical performance indicator for practical applications, 
TABLE 3 | Overall performance of three benchmark algorithms on the testing set.

\begin{tabular}{|c|c|c|c|c|c|}
\hline Algorithm & FN & FP & Sensitivity (\%) & Specificity (\%) & Lead time (ms) \\
\hline Threshold & $20 / 444$ & $84 / 507$ & 95.50 & 83.43 & $333 \pm 160$ \\
\hline SVM & $1 / 444$ & $26 / 507$ & 99.77 & 94.87 & $385 \pm 159$ \\
\hline ConvLSTM & $3 / 444$ & $5 / 507$ & 99.32 & 99.01 & $403 \pm 163$ \\
\hline
\end{tabular}

FN, false alarm; FP, false positive.

TABLE 4 | Validation performance of ConvLSTM on the FARSEEING real-world fall dataset.

\begin{tabular}{|c|c|c|c|c|c|}
\hline Algorithm & FN & FP & Sensitivity (\%) & Specificity (\%) & Lead time (ms) \\
\hline ConvLSTM & $1 / 15$ & $4 / 15$ & 93.33 & 73.33 & $411 \pm 317$ \\
\hline
\end{tabular}

FN, false alarm; FP, false positive.

such as on-demand airbags for fall injury prevention. In such a wearable system, a short lead time may fail to prevent fallinduced injuries since it is too short to fully inflate the airbag before the body-ground impact. In this work, both ConvLSTM $(403 \pm 163 \mathrm{~ms})$ and SVM $(385 \pm 159 \mathrm{~ms})$ showed a much longer lead time than the threshold-based algorithm (333 \pm $160 \mathrm{~ms})$, considering the very short duration of falling $(\sim 746 \mathrm{~ms}$ as reported earlier). This fact also indicates that the features used in ConvLSTM and SVM are more comprehensive and robust to distinguish falls at the beginning stage of falling from ADLs. Readers should be aware that, in this study, only accuracy measures (sensitivity and specificity) and lead time were considered to evaluate the benchmark algorithms. Other practical issues, such as the computational resource and battery capacity in wearable embedded devices, should be explored in the future to have a more comprehensive evaluation of the algorithms (Torti et al., 2018).

It is worth discussing the sampling frequency and location of the sensor used in our KFall dataset. Since KFall is designed for pre-impact fall detection and proactive injury prevention rather than post-fall detection, only part of the fall data can be seen by the pre-impact fall detection algorithms. Considering the short period of falling (average $746 \mathrm{~ms}$, Tao and Yun, 2017) and the buffer time required for the full activation of fall protection devices, such as inflatable airbags, low-frequency sensor data may not provide sufficient motion information and fine details for accurate classification, especially for machine and deep learning algorithms, because they extract features from sliding windows of multiple frames. In a recent review paper on pre-impact fall detection ( $\mathrm{Hu}$ and $\mathrm{Qu}, 2016)$, only three out of 13 studies set the sensor sampling frequency below $100 \mathrm{~Hz}$, and all of these studies applied threshold-based algorithms. Thresholdbased algorithms are less sensitive to the sampling frequency since their working principle is usually based on a single frame of data, not multiple frames. Even for post-fall detection, Saleh et al. (2021) found that the detection accuracy was always improved by increasing the sensor sampling frequency from 20 to $40 \mathrm{~Hz}$ in three different sensor locations. In this study, KFall with a sensor sampling frequency of $100 \mathrm{~Hz}$ achieved promising accuracy and lead time in three benchmark algorithms, which also provides some flexibility for interested readers to evaluate the performance of different algorithms if downsampling is required. Regarding the sensor location, low back was chosen for the KFall dataset due to two main reasons. First, low back has been validated as one of the best sensor positions for fall detection (Ntanasis et al., 2016; Özdemir, 2016), and many studies on pre-impact fall detection have also achieved good accuracy from this position (Shan and Yuan, 2010; Jung et al., 2020). This is understandable since the low back position is close to the center of mass of the human body. Therefore, the motion data collected from this location could represent human motion well. The second reason is related to practical applications of preventing fall-related injuries. Since a hip fracture is one of the most serious fall-related injuries, it can reduce mobility and even cause death (Lord et al., 2007; Jung et al., 2020); the sensor in this location can be easily embedded into a belt-shaped airbag for protecting the hip in real time (Shi et al., 2009; Tamura et al., 2009; Ahn et al., 2018).

There is still an ongoing debate about the effectiveness of applying algorithms trained on simulated fall datasets of the young volunteers to real-world fall detections in the elderly. Klenk et al. (2011) observed that, compared with simulated falls, real-world falls have considerably larger changes in acceleration during the falling phase. While other researchers reported similar features in acceleration signals between two types of falls, they also found that some fall phases detected from simulated falls were not detectable from real falls (Kangas et al., 2012). Bagala et al. (2012) evaluated 13 published threshold-based algorithms on an acceleration dataset with 29 real-world falls. Their results showed the average sensitivity and specificity were 57 and $83 \%$, respectively, which were much worse than the results of detecting simulated falls. On the contrary, another group of researchers trained the SVM algorithm based on their simulated fall dataset and further tested the model on the FARSEEING realworld fall dataset (Chen et al., 2017); they achieved both high sensitivity and specificity ( $>95 \%)$ in detecting real-world falls. The potential reason for this conflicting result may be that the features extracted from windows in machine learning algorithms have more distinguishing power than the features extracted from discrete frames in threshold-based algorithms. However, both studies only focused on post-fall detection because they did not 
investigate whether the fall was detected before the body hit the ground. In this study, we also used the FARSEEING real-world fall dataset to externally validate the best trained ConvLSTM model from our simulated fall dataset (KFall) for pre-impact fall detection. The results showed high sensitivity since 14 out of the 15 real-world falls were successfully detected before body-ground impact (Table 4). However, the specificity dropped sharply compared to the performance in the simulated fall dataset, which could be understandable since only acceleration data were used to train the model due to the lack of angular velocity and orientation data in FARSEEING dataset. Our validation results demonstrated a certain potential of using the simulated dataset for real-world pre-impact fall detection. For better validation, a larger real-world fall dataset with more comprehensive motion signals (including acceleration, angular velocity, and orientation) is needed.

There are some limitations related to our current KFall dataset. First, the current KFall only contains simulated falls from young male adults due to safety concerns and practical convenience. Caution is thus needed to directly apply KFall dataset into real-world applications. Second, the current KFall dataset does not include normal ADLs from older subjects due to some practical limitations from the COVID-19 pandemic. We will further expand our KFall dataset by recruiting older and female subjects as well as evaluate false alarm rates of benchmark algorithms on the target population in the future.

\section{CONCLUSION}

In this paper, we proposed and publicly provided a comprehensive motion dataset called "KFall" for pre-impact fall detection. This new dataset was developed from 32 Korean participants while performing 21 types of ADLs and 15 types of falls. The motion data contain acceleration, angular velocity, and Euler angle, which are collected from a nine-axis inertial sensor attached at the low back of each participant. Compared with the existing public datasets, the advantages of the KFall dataset are 3-fold. First of all, it covers almost all typical ADLs and falls, thus getting closer to the complex real-world scenarios. Secondly but more importantly, the KFall dataset is constructed together with a synchronized video camera at a high frame rate of $90 \mathrm{~Hz}$, which makes it the first public dataset for pre-impact fall detection, not just for post-fall detection. In this process, we further introduced a practical and semiautomatic method to label the fall onset moment by integrating information from the sensor and video data. Thirdly, we also developed

\section{REFERENCES}

Ahn, S., Choi, D., Kim, J., Kim, S., Jeong, Y., Jo, M., et al. (2018). Optimization of a pre-impact fall detection algorithm and development of hip protection airbag system. Sens. Mater. 30, 1743-1752. doi: 10.18494/SAM.2018.1876

Aziz, O., Musngi, M., Park, E. J., Mori, G., and Robinovitch, S. N. (2017). A comparison of accuracy of fall detection algorithms (threshold-based vs. machine learning) using waist-mounted tri-axial accelerometer signals from a three different types of state-of-the-art algorithms (threshold based, a support-vector machine, and deep learning), using the KFall dataset for pre-impact fall detection. Performance of these algorithms could be regarded as benchmarks for further developing better algorithms with this new dataset. This large-scale motion dataset and benchmark algorithms could provide researchers and practitioners valuable data and references to develop new technologies and strategies for pre-impact fall detection and proactive injury prevention for the elderly.

\section{DATA AVAILABILITY STATEMENT}

The datasets presented in this study can be found in online repositories. The names of the repository/repositories and accession number(s) can be found in the article/supplementary material.

\section{ETHICS STATEMENT}

The studies involving human participants were reviewed and approved by KAIST Institutional Review Board. The patients/participants provided their written informed consent to participate in this study.

\section{AUTHOR CONTRIBUTIONS}

SX conceptualized the study, obtained the funding, and reviewed the edited manuscript. XY developed the data acquisition software, developed the benchmark algorithms, and wrote the original draft. XY and JJ conducted the experiment. All authors contributed to the article and approved the submitted version.

\section{FUNDING}

This work was supported by the Basic Science Research Program through the National Research Foundation of Korea funded by the Ministry of Science, ICT and Future Planning (NRF- 2017R1C1B2006811), and the KAIST Faculty Research Fund (A0601003029).

\section{ACKNOWLEDGMENTS}

We thank HueiEe Yap for his technical support on the sensor data acquisition and the FARSEENING consortium for the use of their fall repository. We also thank all the subjects who participated in this study. 
Bagala, F., Becker, C., Cappello, A., Chiari, L., Aminian, K., Hausdorff, J. M., et al. (2012). Evaluation of accelerometer-based fall detection algorithms on real-world falls. PLoS ONE 7:e37062. doi: 10.1371/journal.pone.0037062

Bourke, A., Van De Ven, P., Gamble, M., O'connor, R., Murphy, K., Bogan, E., et al. (2010). Evaluation of waist-mounted tri-axial accelerometer based falldetection algorithms during scripted and continuous unscripted activities. J. Biomech. 43, 3051-3057. doi: 10.1016/j.jbiomech.2010.07.005

Bourke, A. K., O'donovan, K. J., and Olaighin, G. (2008). The identification of vertical velocity profiles using an inertial sensor to investigate pre-impact detection of falls. Med. Eng. Phys. 30, 937-946. doi: 10.1016/j.medengphy.2007.12.003

Casilari, E., Lora-Rivera, R., and García-Lagos, F. (2020). A study on the application of convolutional neural networks to fall detection evaluated with multiple public datasets. Sensors 20:1466. doi: 10.3390/s20051466

Casilari, E., Santoyo-Ramón, J. A., and Cano-García, J. M. (2017a). Analysis of public datasets for wearable fall detection systems. Sensors (Switzerland) 17:71513. doi: $10.3390 / \mathrm{s} 17071513$

Casilari, E., Santoyo-Ramón, J. A., and Cano-García, J. M. (2017b). "UMAFall: a multisensor dataset for the research Q18 on automatic fall detection," in Procedia Computer Science, eds E. Shakshuki and A. Yasar (Leuven: Elsevier), 32-39. doi: 10.1016/j.procs.2017.06.110

Chen, K. H., Hsu, Y. W., Yang, J. J., and Jaw, F. S. (2017). Enhanced characterization of an accelerometer-based fall detection algorithm using a repository. Instrum. Sci. Techn. 45, 382-391. doi: 10.1080/10739149.2016.1268155

Frank, K., Vera Nadales, M. J., Robertson, P., and Pfeifer, T. (2010). "Bayesian recognition of motion related activities with inertial sensors," in Proceedings of the 12th ACM International Conference Adjunct Papers on Ubiquitous Computing-Adjunct, 445-446. doi: 10.1145/1864431.1864480

Gasparrini, S., Cippitelli, E., Gambi, E., Spinsante, S., Wåhslén, J., Orhan, I., et al. (2015). "Proposal and experimental evaluation of fall detection solution based on wearable and depth data fusion," in International Conference on ICT Innovations (Ohrid: Springer), 99-108. doi: 10.1007/978-3-319-25733-4_11

$\mathrm{Hu}, \mathrm{X}$., and Qu, X. (2016). Pre-impact fall detection. Biomed. Eng. Online 15, 1-16. doi: 10.1186/s12938-016-0194-X

Igual, R., Medrano, C., and Plaza, I. (2013). Challenges, issues and trends in fall detection systems. Biomed. Eng. Online 12, 1-24. doi: 10.1186/1475-925X-12-66

Incel, O. D. (2015). Analysis of movement, orientation and rotation-based sensing for phone placement recognition. Sensors 15, 25474-25506. doi: $10.3390 / \mathrm{s} 151025474$

Jung, H., Koo, B., Kim, J., Kim, T., Nam, Y., and Kim, Y. (2020). Enhanced algorithm for the detection of preimpact fall for wearable airbags. Sensors 20:1277. doi: 10.3390/s20051277

Kangas, M., Vikman, I., Nyberg, L., Korpelainen, R., Lindblom, J., and Jäms,ä, T. (2012). Comparison of real-life accidental falls in older people with experimental falls in middle-aged test subjects. Gait Posture 35, 500-505. doi: 10.1016/j.gaitpost.2011.11.016

Kim, T. H., Choi, A., Heo, H. M., Kim, K., Lee, K., and Mun, J. H. (2019). Machine learning-based pre-impact fall detection model to discriminate various types of fall. J. Biomech. Eng. 141:43449. doi: 10.1115/1.4043449

Kim, Y., Jung, H., Koo, B., Kim, J., Kim, T., and Nam, Y. (2020). Detection of preimpact falls from heights using an inertial measurement unit sensor. Sensors 20:5388. doi: 10.3390/s20185388

Klenk, J., Becker, C., Lieken, F., Nicolai, S., Maetzler, W., Alt, W., et al. (2011). Comparison of acceleration signals of simulated and real-world backward falls. Med. Eng. Phys. 33, 368-373. doi: 10.1016/j.medengphy.2010.11.003

Klenk, J., Schwickert, L., Palmerini, L., Mellone, S., Bourke, A., Ihlen, E., et al. (2016). The FARSEEING real-world fall repository: a large-scale collaborative database to collect and share sensor signals from real-world falls. Europ. Rev. Aging Phys. Activity 13:8. doi: 10.1186/s11556-016-0168-9

Kwolek, B., and Kepski, M. (2014). Human fall detection on embedded platform using depth maps and wireless accelerometer. Comput. Methods Programs Biomed. 117, 489-501. doi: 10.1016/j.cmpb.2014.09.005

Lee, S., Hong, S., and Jung, S. (2014). "Estimation for the vertical speed of helicopter using MEMS inertial sensor," in Proceeding of Autumn Conference of The Korean Society For Aeronautical And Space Sciences (in Korean) (Wonju), 1422-1425.
Lord, S. R., Sherrington, C., Menz, H. B., and Close, J. C. T. (2007). Falls in Older People: Risk Factors and Strategies for Prevention. Cambridge: Cambridge University Press. doi: 10.1017/CBO9780511722233

Martínez-Villaseñor, L., Ponce, H., Brieva, J., Moya-Albor, E., Núñez-Martínez, J., and Peñafort-Asturiano, C. (2019). Up-fall detection dataset: a multimodal approach. Sensors (Switzerland) 19:1988. doi: 10.3390/s19091988

Medrano, C., Igual, R., Plaza, I., and Castro, M. (2014). Detecting falls as novelties in acceleration patterns acquired with smartphones. PLoS ONE 9:e94811. doi: 10.1371/journal.pone.0094811

Micucci, D., Mobilio, M., and Napoletano, P. (2017). Unimib shar: a dataset for human activity recognition using acceleration data from smartphones. Appl. Sci. 7:1101. doi: 10.3390/app7101101

Musci, M., De Martini, D., Blago, N., Facchinetti, T., and Piastra, M. (2020). Online Fall Detection Using Recurrent Neural Networks on Smart Wearable Devices. IEEE Transactions on Emerging Topics in Computing. doi: 10.1109/TETC.2020.3027454

Noury, N., Rumeau, P., Bourke, A., laighin, G., and Lundy, J. (2008). A proposal for the classification and evaluation of fall detectors. Irbm 29, 340-349. doi: 10.1016/j.irbm.2008.08.002

Ntanasis, P., Pippa, E., zdemir, A. T., Barshan, B., and Megalooikonomou, V. (2016). "Investigation of sensor placement for accurate fall detection," in International Conference on Wireless Mobile Communication and Healthcare (Milan: Springer), 225-232. doi: 10.1007/978-3-319-58877-3_30

Ojetola, O., Gaura, E., and Brusey, J. (2015). "Data set for fall events and daily activities from inertial sensors," in Proceedings of the 6th ACM Multimedia Systems Conference (Portland, OR), 243-248. doi: 10.1145/2713168.2713198

Organization, W. H., Ageing, W. H. O., and Unit, L. C. (2008). WHO Global Report on Falls Prevention in Older Age. World Health Organization.

Özdemir, A.T. (2016). An analysis on sensor locations of the human body for wearable fall detection devices: principles and practice. Sensors 16:1161. doi: 10.3390/s16081161

Petersen, K. (2020). LPMS User Manual. Available online at: https://lp-research. atlassian.net/wiki/spaces/LKB/pages/1100611599/LPMS\$+\$User\$+\$Manual\# Orientation-Data (accessed December 15, 2020).

Qiu, H., Rehman, R. Z. U., Yu, X., and Xiong, S. (2018). Application of wearable inertial sensors and a new test battery for distinguishing retrospective fallers from non-fallers among community-dwelling older people. Scient. Rep. 8, 1-10. doi: 10.1038/s41598-018-34671-6

Sabatini, A. M., Ligorio, G., Mannini, A., Genovese, V., and Pinna, L. (2015). Prior-to-and post-impact fall detection using inertial and barometric altimeter measurements. IEEE Trans. Neural Syst. Rehab. Eng. 24, 774-783. doi: 10.1109/TNSRE.2015.2460373

Saha, S. S., Rahman, S., Rasna, M. J., Islam, A. M., and Ahad, M.,a.R. (2018). "DU-MD: An open-source human action dataset for ubiquitous wearable sensors," in 2018 Joint 7th International Conference on Informatics, Electronics \& Vision (ICIEV) and 2018 2nd International Conference on Imaging, Vision \& Pattern Recognition (icIVPR) (Kitakyushu: IEEE), 567-572. doi: 10.1109/ICIEV.2018.86 41051

Saleh, M., Abbas, M., and Le Jeannes, R. B. (2021). FallAllD: an open dataset of human falls and activities of daily living for classical and deep learning applications. IEEE Sensors J. 21, 1849-1858. doi: 10.1109/JSEN.2020.3018335

Shan, S., and Yuan, T. (2010). "A wearable pre-impact fall detector using feature selection and support vector machine," in IEEE 10th International Conference On Signal Processing Proceedings (Beijing: IEEE), 1686-1689. doi: 10.1109/ICOSP.2010.5656840

Shi, G., Chan, C. S., Li, W. J., Leung, K.-S., Zou, Y., and Jin, Y. (2009). Mobile human airbag system for fall protection using MEMS sensors and embedded SVM classifier. IEEE Sensors J. 9, 495-503. doi: 10.1109/JSEN.2008.2012212

Shi, Y., Shi, Y., and Wang, X. (2012). "Fall detection on mobile phones using features from a five-phase model," in 2012 9th International Conference on Ubiquitous Intelligence and Computing and 9th International Conference on Autonomic and Trusted Computing (Fukuoka: IEEE), 951-956. doi: 10.1109/UIC-ATC.2012.100

Sucerquia, A., López, J. D., and Vargas-Bonilla, J. F. (2017). SisFall: a fall and movement dataset. Sensors (Switzerland) 17:198. doi: 10.3390/s17010198 
Tamura, T., Yoshimura, T., Sekine, M., Uchida, M., and Tanaka, O. (2009). A wearable airbag to prevent fall injuries. IEEE Trans. Inform. Techn. Biomed. 13, 910-914. doi: 10.1109/TITB.2009.2033673

Tao, X., and Yun, Z. (2017). Fall prediction based on biomechanics equilibrium using Kinect. Int. J. Distrib. Sensor Netw. 13:1550147717703257. doi: $10.1177 / 1550147717703257$

Torti, E., Fontanella, A., Musci, M., Blago, N., Pau, D., Leporati, F., et al. (2018). "Embedded real-time fall detection with deep learning on wearable devices," in 2018 21st Euromicro Conference on Digital System Design (DSD) (Prague: IEEE), 405-412. doi: 10.1109/DSD.2018.00075

Tran, T. H., Le, T. L., Pham, D. T., Hoang, V. N., Khong, V. M., Tran, Q. T., et al. (2018). "A multi-modal multi-view dataset for human fall analysis and preliminary investigation on modality," in Proceedings - International Conference on Pattern Recognition (Beijing), 1947-1952. doi: 10.1109/ICPR.2018.8546308

Vavoulas, G., Chatzaki, C., Malliotakis, T., Pediaditis, M., and Tsiknakis, M. (2016). "The MobiAct dataset: recognition of activities of daily living using smartphones," in ICT4AgeingWell (Rome), 143-151. doi: 10.5220/0005792401430151

Vavoulas, G., Pediaditis, M., Chatzaki, C., Spanakis, E. G., and Tsiknakis, M. (2014). The mobifall dataset: fall detection and classification with a smartphone. Int. J. Monit. Surveill. Techn. Res. 2, 44-56. doi: 10.4018/ijmstr.2014010103

Wang, F.-T., Chan, H.-L., Hsu, M.-H., Lin, C.-K., Chao, P.-K., and Chang, Y.-J. (2018). Threshold-based fall detection using a hybrid of tri-axial accelerometer and gyroscope. Physiol. Measur. 39:105002. doi: 10.1088/1361-6579/aae0eb

Wang, J., Chen, Y., Hao, S., Peng, X., and Hu, L. (2019). Deep learning for sensor-based activity recognition: a survey. Pattern Recogn. Lett. 119, 3-11. doi: 10.1016/j.patrec.2018.02.010

Wang, L., Peng, M., and Zhou, Q. (2020). Pre-impact fall detection based on multi-source CNN ensemble. IEEE Sensors J. 20, 5442-5451. doi: 10.1109/JSEN.2020.2970452
Wu, Y., Su, Y., Feng, R., Yu, N., and Zang, X. (2019). Wearable-sensor-based preimpact fall detection system with a hierarchical classifier. Measurement 140, 283-292. doi: 10.1016/j.measurement.2019.04.002

$\mathrm{Yu}$, S., Chen, H., and Brown, R. A. (2017). Hidden Markov model-based fall detection with motion sensor orientation calibration: a case for real-life home monitoring. IEEE J. Biomed. Health Inform. 22, 1847-1853. doi: 10.1109/JBHI.2017. 2782079

Yu, X., Jang, J., and Xiong, S. (2021). "Machine learning-based pre-impact fall detection and injury prevention for the elderly with wearable inertial sensors," in International Conference on Applied Human Factors and Ergonomics New York, NY: Springer), 1-8.

Yu, X., Qiu, H., and Xiong, S. (2020). A novel hybrid deep neural network to predict pre-impact fall for older people based on wearable inertial sensors. Front. Bioeng. Biotech. 8:63. doi: 10.3389/fbioe.2020. 00063

Zhao, G., Mei, Z., Liang, D., Ivanov, K., Guo, Y., Wang, Y., et al. (2012). Exploration and implementation of a pre-impact fall recognition method based on an inertial body sensor network. Sensors 12, 15338-15355. doi: $10.3390 / \mathrm{s} 121115338$

Conflict of Interest: The authors declare that the research was conducted in the absence of any commercial or financial relationships that could be construed as a potential conflict of interest.

Copyright (c) 2021 Yu, Jang and Xiong. This is an open-access article distributed under the terms of the Creative Commons Attribution License (CC BY). The use, distribution or reproduction in other forums is permitted, provided the original author(s) and the copyright owner(s) are credited and that the original publication in this journal is cited, in accordance with accepted academic practice. No use, distribution or reproduction is permitted which does not comply with these terms. 